Article

\title{
The Analysis of the Aerodynamic Character and Structural Response of Large-Scale Wind Turbine Blades
}

\author{
Xin Cai ${ }^{1,2}$, Pan Pan ${ }^{1, *}$, Jie Zhu ${ }^{1}$ and Rongrong Gu ${ }^{1}$ \\ 1 College of Mechanics and Materials, Hohai University, Nanjing 210098, China; \\ E-Mails: xcai@hhu.edu.cn (X.C.); zhukejie2222@163.com (J.Z.); gurr99@126.com (R.G.) \\ 2 College of Water Conservancy and Hydropower Engineering, Hohai University, \\ Nanjing 210098, China \\ * Author to whom correspondence should be addressed; E-Mail: panpan.159@163.com; \\ Tel.: +86-159-5057-9343; Fax: +86-519-8510-3280.
}

Received: 22 April 2013; in revised form: 20 June 2013 / Accepted: 20 June 2013 /

Published: 27 June 2013

\begin{abstract}
A process of detailed CFD and structural numerical simulations of the $1.5 \mathrm{MW}$ horizontal axis wind turbine (HAWT) blade is present. The main goal is to help advance the use of computer-aided simulation methods in the field of design and development of HAWT-blades. After an in-depth study of the aerodynamic configuration and materials of the blade, 3-D mapping software is utilized to reconstruct the high fidelity geometry, and then the geometry is imported into CFD and structure finite element analysis (FEA) software for completely simulation calculation. This research process shows that the CFD results compare well with the professional wind turbine design and certification software, GH-Bladed. Also, the modal analysis with finite element method (FEM) predicts well compared with experiment tests on a stationary blade. For extreme wind loads case that by considering a 50-year extreme gust simulated in CFD are unidirectional coupled to the FE-model, the results indicate that the maximum deflection of the blade tip is less than the distance between the blade tip (the point of maximum deflection) and the tower, the material of the blade provides enough resistance to the peak stresses the occur at the conjunction of shear webs and center spar cap. Buckling analysis is also included in the study.
\end{abstract}

Keywords: rotational effect; fluid structure interaction; eigenbuckling; Mieses stress 


\section{Introduction}

To capture higher wind speeds, wind turbines have increased in height and size and this reduces the number of individual turbine units possible on a wind farm and in turn the cost of operation of the farm. For example, the first $5 \mathrm{MW}$ offshore wind turbine unit with a rotor diameter of $115 \mathrm{~m}$, and a hub height reaching more than $100 \mathrm{~m}$ has been recently installed in the Fujian Province of China.

To design and develop more efficient and reliable wind turbines, the way to accurately predict aerodynamic characteristics and structure response is of critical significance. There are various classical aerodynamic models available in the literature: The blade element momentum (BEM) method could be a design as well as a verification tool which splits the blade into small elements, airfoil data of each element is provided for lift and drag coefficients as a function of angle of attack, so performance of the turbine can be calculated by summing the contribution of all the elements [1]; to obtain more aerodynamic characteristics of wind turbines, studies of the wake configuration have been likewise been conducted. The prescribed-vortex wake [2], free-vortex wake method [3] as well as lifting panel method [4] show good application prospects. In recent years, advances in computer technology that enabled computational fluid dynamics (CFD) techniques to deal with more aerodynamic issues, like boundary layer transition, dynamic stall, inflow turbulence, rotational effects, and so on. On the other hand, CFD analysis of wind turbines can be performed at a lesser cost than that required to set up a wind tunnel and perform full scale experiments. There has been a growing use of CFD codes over the last decade for turbine blade performance analysis, among them the ANSYS CFX software has a good reputation for its good performance on turbo-machinery simulation, as well as aeroelasticity analysis $[5,6]$.

To investigate the global behaviors in terms of, for example, eigenfrequencies, tip deflections, buckling, and global stress/strain levels of wind turbine blades at certain wind speeds, finite element methods (FEM) have gained wider acceptance as they have become more capable in their predictive capability. In [7], Jensen used FE-code MSC-Marc to simulate the Brazier effect of the load-carrying box girder of a $34 \mathrm{~m}$ composite blade. Hermann [8] and Raiauurai [9] applied ANSYS software to simulate the buckling and free vibration behavior of wind turbine blades, respectively. Grujicic [10,11] used ABAQUS/Standard to calculate the equivalent strain and Von Mises distribution over the large-scale HAWT-blade caused by a $70 \mathrm{~m} / \mathrm{s}$ gust.

Rotor blade shells are made of monolithic composite laminates in the root and main spar areas, and the other parts include webs constructed with sandwich composites. However, the laminates are assembled of various fibrous layers in the entire blade and vary in thickness along the longitudinal as well as the transverse direction due to the "ply drop" [12]. In order to predict the global behaviors accurately, intensive effort must be devoted to investigate the material properties, the start and end position, the amount and sequence of the various layers of the assigned laminates.

The main objective of this paper is to adopt commercial software, like CFX and ANSYS, to simulate a wind turbine blade which has already been installed and put into operation under certain conditions, and compare these results to experimental tests and some international certification standards. It is hoped that a set of computer-aided approaches could be put forward in the wind turbine blades design/development area so that many critical decisions and modifications can be made to improve the quality of large-scale HAWT-blades. 
The paper is organized as follows: Section 2 presents detailed specifications of the $1.5 \mathrm{MW}$ HAWT-blade. Section 3 presents the CFD simulation and aerodynamics characteristics of this blade. Section 4 presents the accurate FE-model constructing and dynamic characteristics of this blade, and then its static behaviors under limit loads obtained in Section 3 are calculated. The simulation results provide several suggestions for further modification of large-scale blades. Section 5 summaries the key conclusions resulting from the present work.

\section{Specifications of 1.5 MW Wind Turbine Blade}

The rotor blade investigated in this paper is designed for a 3-blade upwind wind turbine, which is nominally $37.5 \mathrm{~m}$ long and controlled by the pitch-regulated variable speed method. The thickness-to-chord ratio $(t / c)$ of the airfoils chosen for the blade varies from $40 \%$ in the blade shoulder $(r / R=0.215)$ to $18 \%$ in the blade tip. The difference between the maximum and minimum twist angle is $8.78^{\circ}$. The diameter of the cylindrical blade root is $1.8 \mathrm{~m}$, and the length of the cord in the blade shoulder is $3.08 \mathrm{~m}$. The specific configurations of the blade are summarized in Table 1.

Table 1. Specifications of the $1.5 \mathrm{MW}$ wind turbine blade.

\begin{tabular}{cc}
\hline Parameters & Value \\
\hline Rated wind speed $(\mathrm{m} / \mathrm{s})$ & 11.5 \\
Rated rotor speed $(\mathrm{rpm})$ & 19.0 \\
Cut in wind speed $(\mathrm{m} / \mathrm{s})$ & 3.5 \\
Cut out wind speed $(\mathrm{m} / \mathrm{s})$ & 25.0 \\
Extreme wind speed $(\mathrm{m} / \mathrm{s})$ & 59.0 \\
Rotor overhang $(\mathrm{m})$ & 4.2 \\
\hline
\end{tabular}

\section{Aerodynamic Analysis}

In order to maintain the same aerodynamic configuration fidelity as the initial design, the 3D-modeling software UG NX is utilized to compile the global point coordinates that define the airfoil profile of the blade's each section. For this purpose about 70 points are used for the suction side of the airfoil and about 80 for the pressure side, which are subsequently connected via a spline curve. Each surface between two sections is created through four lines, then, the aerodynamic configuration with solid body is created by suturing all the surfaces, which is shown in Figure 1.

Figure 1. Aerodynamic configuration of the $1.5 \mathrm{MW}$ blade.

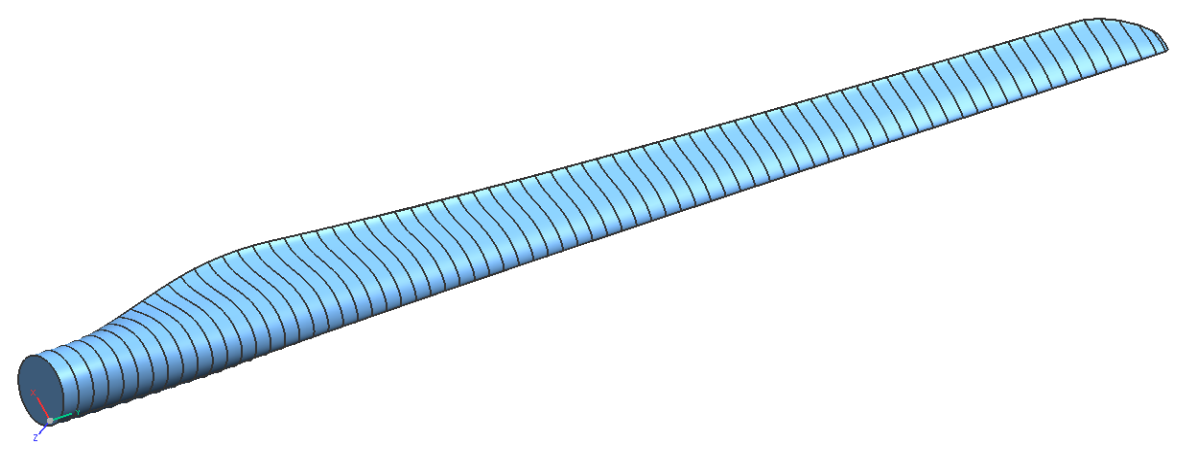




\subsection{Simulation Method}

In the present work, only one of the three blades is explicitly modeled in the computations. The remaining blades are accounted for using periodic boundary conditions, exploiting the $120^{\circ}$ symmetry of the three-bladed rotor, therefore, a $1 / 3$ cylindrical numerical wind tunnel is constructed in the UG NX. The numerical tunnel consists of two domains, namely the rotational domain which contains the blade, and the stationary domain. The rotational domain is defined by the reference length of the rotor radius of $39 \mathrm{~m}$. A spatial resolution of $102 \mathrm{~m}$ to the calculation domain inlet, $340 \mathrm{~m}$ to the downstream region, and $140 \mathrm{~m}$ to the radial direction of the wind tunnel is applied.

The tunnel geometrical model composite of stationary and rotational domain is then imported into ICEM software, and the two domains are meshed with hexahedral grid respectively. The overall structured grid is shown in Figure 2a.

Around the blade, the O-grid block topology is used twice. First, the outer O type blocks are generated as the nearfield grids to cover the rotor blade. Second, the inner O-type blocks are generated to overlap the blade surface, and the blocks filled in the blade are deleted completely. During the creation of the mesh, great care must be taken at the vicinity close to the blade surface since it is where the boundary layer will be formed, therefore, the first height in the normal direction from the surface of the rotor blade is about $0.2 \mathrm{~mm}$ which is defined by a target $\mathrm{y}^{+}$value, given the Reynolds Number and a Reference Length, and the spacing ratio is 1.1 in the normal direction. 21 nodes are meshed in the first $\mathrm{O}$ type block, as can be seen in Figure $2 \mathrm{~b}$ which shows the quad meshes around the radial station located at $r / R=0.58$. The total cell number of the numerical grid is about $\mathrm{N}=4.5 \times 10^{6}$, consisting fully of hexahedral grids in the whole domains.

Figure 2. (a) Structured hexahedral grid of total domain. (b) Quad meshes around the airfoil.

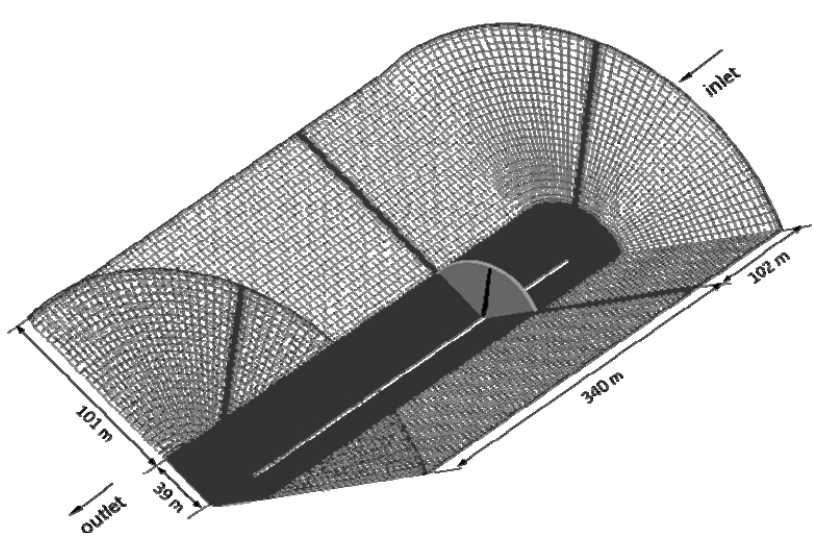

(a)

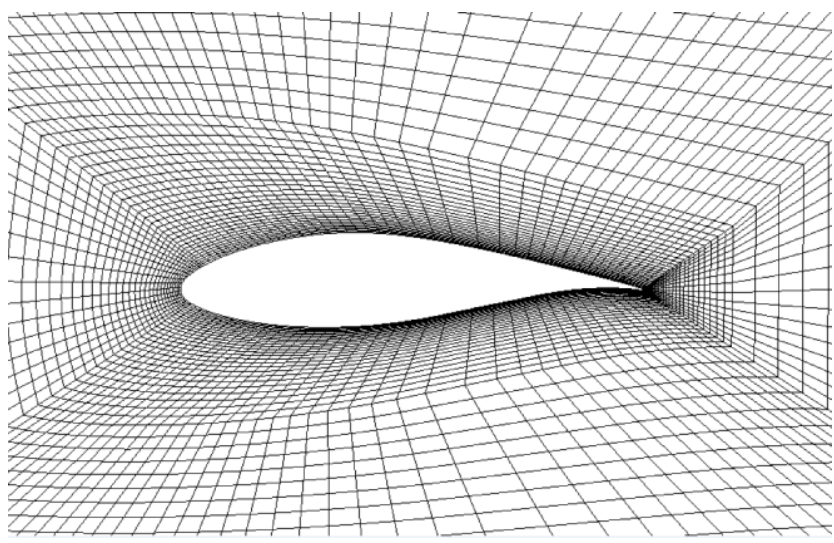

(b)

The CFD calculations are run in the ANSYS CFX 12.1 environment. In this paper, the turbulence in the boundary layer is modeled by the Shear-Stress Transport (SST) model. The model is chosen because the $k-\omega$ based SST model accounts for the transport of the turbulent shear and gives highly accurate predictions of the onset and the amount of flow separation under adverse pressure gradient [13]. The complete formulation of the SST model is given below: 


$$
\left\{\begin{array}{c}
\frac{\partial(\rho k)}{\partial t}+\frac{\partial\left(\rho U_{i} k\right)}{\partial x_{i}}=\tilde{P}_{k}-\beta^{*} \rho k \omega+\frac{\partial}{\partial x_{i}}\left[\left(\mu+\sigma_{k} \mu_{t}\right) \frac{\partial k}{\partial x_{i}}\right] \\
\frac{\partial(\rho \omega)}{\partial t}+\frac{\partial\left(\rho U_{i} \omega\right)}{\partial x_{i}}=\alpha \rho S^{2}-\beta \rho \omega^{2}+\frac{\partial}{\partial x_{i}}\left[\left(\mu+\sigma_{\omega} \mu_{t}\right) \frac{\partial \omega}{\partial x_{i}}\right]+2\left(1-F_{1}\right) \rho \sigma_{\omega 2} \frac{1}{\omega} \frac{\partial k}{\partial x_{i}} \frac{\partial \omega}{\partial x_{i}}
\end{array}\right.
$$

where the blending function $F_{1}$ is defined by:

$$
F_{1}=\tanh \left\{\left\{\min \left[\max \left(\frac{\sqrt{k}}{\beta^{*} \omega y}, \frac{500 v}{y^{2} \omega}\right), \frac{4 \rho \sigma_{\omega 2} k}{C D_{k \omega} y^{2}}\right]\right\}^{4}\right\}
$$

where $C D_{k \omega}=\max \left(2 \rho \sigma_{\omega 2} \frac{1}{\omega} \frac{\partial k}{\partial x_{i}} \frac{\partial \omega}{\partial x_{i}}, 10^{-10}\right) ; y$ is the distance to the nearest wall; $v$ is the kinematic viscosity.

$F_{1}$ is equal to zero away from the surface ( $k-\varepsilon$ model), and switches over to one inside the boundary layer $(k-\omega$ model $)$.

The proper transport behavior can be obtained by a limiter to the formulation of the eddy-viscosity, which is defined as follows:

$$
v_{t}=\frac{a_{1} k}{\max \left(a_{1} \omega, S F_{2}\right)}
$$

where $S$ is the invariant measure of the strain rate and $F_{2}$ is a second blending function defined by:

$$
F_{2}=\tanh \left[\left[\max \left(\frac{2 \sqrt{k}}{\beta^{*} \omega y}, \frac{500 v}{y^{2} \omega}\right)\right]^{2}\right]
$$

A production limiter is used in the SST model to prevent the build-up of turbulence in stagnation regions:

$$
P_{k}=\mu_{t} \frac{\partial U_{i}}{\partial x_{j}}\left(\frac{\partial U_{i}}{\partial x_{j}}+\frac{\partial U_{j}}{\partial x_{i}}\right) \rightarrow \quad \tilde{P}_{k}=\min \left(P_{k}, 10 \beta^{*} \rho k \omega\right)
$$

All constants are computed by a blend from the corresponding constants of the $k-\varepsilon$ and the $k-\omega$ model via $\alpha=\alpha_{1} F+\alpha_{2}(1-F)$. The constants for this model are: $\beta^{*}=0.09 ; \alpha_{1}=5 / 9 ; \beta_{1}=3 / 40$; $\sigma_{k 1}=0.85 ; \sigma_{\omega 1}=0.5 ; \alpha_{2}=0.44 ; \beta_{2}=0.0828 ; \sigma_{k 2}=1 ; \sigma_{\omega 2}=0.856$.

For the boundary conditions, a normal speed condition with a lower turbulent intensity of $14 \%$ which is defined in IEC 61400-1 standard [14] is applied at the inlet boundary, and an average static pressure condition is applied at the outlet condition. No-slip condition is selected for the blade, nacelle and outer domain surfaces. The periodic conditions are applied at the $120^{\circ}$ cyclic boundaries.

For convergence control, because the meshes are refined nearby the blade surface, convergence issues will arise since smaller flow features such as shedding phenomena will be resolved effectively, which coarser meshes cannot capture. The value of the root mean square (RMS) normalized residual over the whole domain is chosen to be below $10^{-4}$, and the maximum amount of iterations is set large enough to monitor the blade torque until stabilization.

Before the CFD results are drawn, the grid-independent analysis is conducted. According to Cimbala's paper [15], the number of nodes of every block's edges is increased by $20 \%$, while the node 
number in the spanwise direction of the blade remains unchanged. After being simulated with the above methods under rated conditions in steady state, the monitored torque doesn't change appreciably compared with initial mesh (error is within $2 \%$ ), so the original grid is adequate.

\subsection{CFD Results and Discussion}

In this paper, six different inflow velocities, $V_{\infty}=5,6.5,8,9.5,10.5,11.5,25 \mathrm{~m} / \mathrm{s}$ are chosen for rotor blade simulation in steady state, meanwhile, the pitch and torque controls are not considered. The contour variations of fluid tangential forces which produce torque on the suction surface of the rotor blade at three wind speeds $(5,11.5,25 \mathrm{~m} / \mathrm{s})$ are shown in Figure 3 . It is observed that the tangential forces mainly concentrate at the outboard stations between $r / R=0.5 \sim 0.8$ at lower wind speed, so the rotor blade is not at its best to produce the maximum power coefficient. At the rated wind speed, the tangential forces contour which appears towards the leading edge and can be observed almost from blade tip to root illustrate that the rotor blade has good aerodynamic performance when pitched at $0^{\circ}$. At $25 \mathrm{~m} / \mathrm{s}$, the blade is in deep stall, the calculated tangential force magnitude is in large scale as well as the axial thrust. Therefore, the active control system should execute collective pitching or individual pitching operation to reduce the angle of attack and hence the lift coefficient.

Figure 3. Fluid tangential forces contour on the suction side.

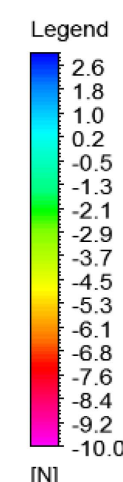

(a) $5 \mathrm{~m} / \mathrm{s}$

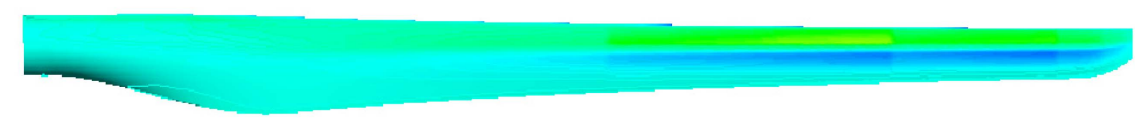

(b) $11.5 \mathrm{~m} / \mathrm{s}$

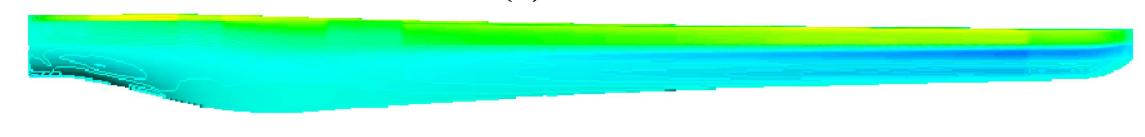

(c) $25 \mathrm{~m} / \mathrm{s}$

The power extraction from air and coefficient are defined in Equations (6) and (7):

$$
\begin{gathered}
\text { Power }=B \cdot T \cdot n \cdot 2 \pi / 60 \\
C_{P}=\frac{\text { Power }}{0.5 \cdot \rho \cdot V_{\infty}^{3} \cdot A}
\end{gathered}
$$

where $B$ is the total number of blades; $T$ is the torque of single blade; $n$ is the rotor speed of wind turbine; $\rho$ is the air density; $A$ is the rotor swept area; and $V_{\infty}$ is the flow velocity.

Figure 4 shows a comparison of the power coefficient computed by GH-Bladed and ANSYS CFX in this paper. As shown from the comparison, $C_{p}$ predicted by CFD agrees well with GH-Bladed at lower wind speed, however, as wind speed rises, CFD predicts lower values than GH-Bladed. Generally, it is true that SST model has excellent prediction ability for wall characteristics, but it 
usually underestimates the rotor torque in the stalled regime which would become evident as wind speed rises.

Figure 4. Power coefficient comparison between CFX and GH-Bladed.

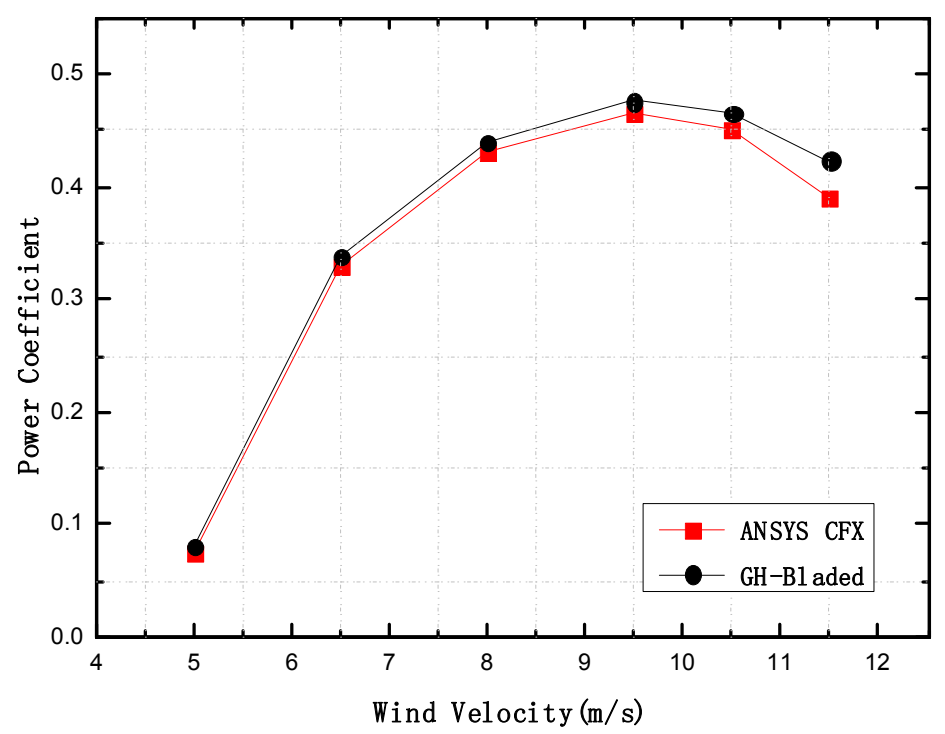

\subsection{Rotational Effects}

For the purpose of applying the BEM method, the stations of the blade are extracted at the interval of $1 \mathrm{~m}$. Each station is computed with 2-D CFD and determined the values of lift coefficient $C_{l}$ and drag coefficient $C_{d}$ as function of attack angle. The power calculated by BEM is $1.762 \mathrm{MW}$, and the axial thrust value is $306.60 \mathrm{kN}$, both are larger than CFD code (the values computed by CFD are $1.513 \mathrm{MW}$ and $280.38 \mathrm{kN}$ ). In Figure 5, the radial stations located at $r / R=0.3,0.6,0.85$ are chosen for pressure comparison evaluated with 3-D and 2-D CFD in the rated condition, the airfoil profile form of the three radial stations can be clearly seen in Figure 6. In the 2-D case, the aerodynamic load of each radial station is computed with twist angle settled the same as its position in blade in the global coordinates. By picking up the axial induction factor $a$ and angular induction factor $a^{\prime}$ of each station with BEM method, the equivalent speed input in 2-D simulation is defined in Equation (8):

$$
V_{r e l}=V_{\infty} \sqrt{(1-a)^{2}+\left(\frac{r \Omega_{r}}{V_{\infty}}\left(1+a^{\prime}\right)\right)^{2}}
$$

where $r$ is the radial station radius; $\Omega_{r}$ is the angular velocity.

It can be seen that the distance between suction and pressure side, i.e., the area of the graph, looks considerably larger in the 2-D case in the figure. Thus, a higher thrust is obtained when the BEM method applied at the design point. At $r / R=0.3$, the 2-D curve falls abruptly, whereas the 3-D trend is smoother after the peak pressure on suction side. The pressure gradient of the flow has a significant effect on the boundary layer, as illustrated in Figure 6, where the in-plane streamlines around the rotating radial station and the corresponding pure 2-D stations are plotted. It is visible that the flow in the boundary layer stops and reverses direction due to the rapid change pressure gradient in the 2-D case. The separating flows from the station place it in stall condition, however, the main effects of rotation are to stabilize vortex shedding and limit the growth of the separation layer, as seen in the 3-D case. 
Figure 5. Comparison of pressure computed by 3-D and 2-D CFD. (a) $r / R=0.3$; (b) $r / R=0.6$; and (c) $r / R=0.85$.

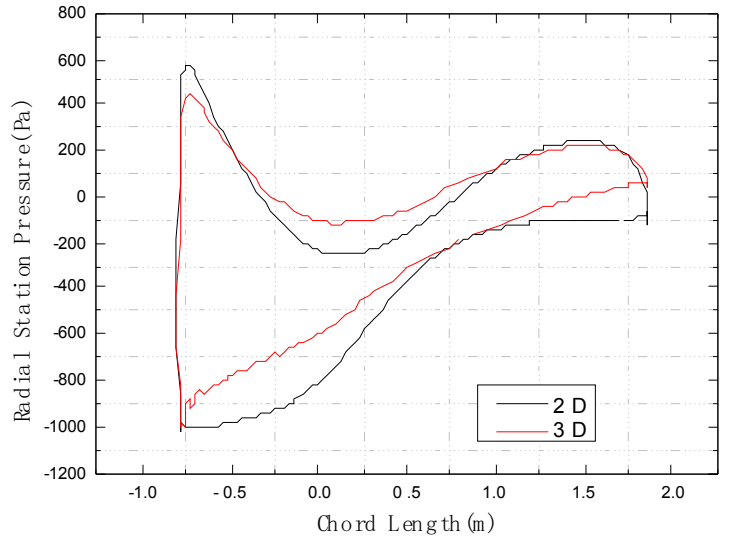

(a)

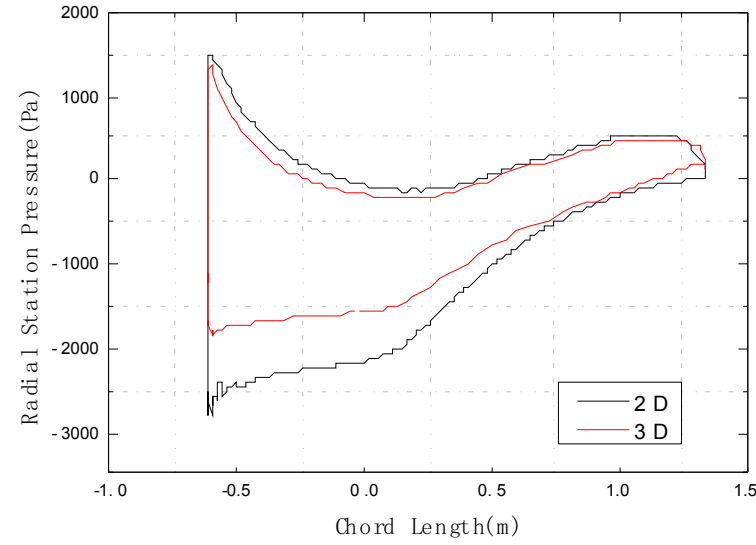

(b)

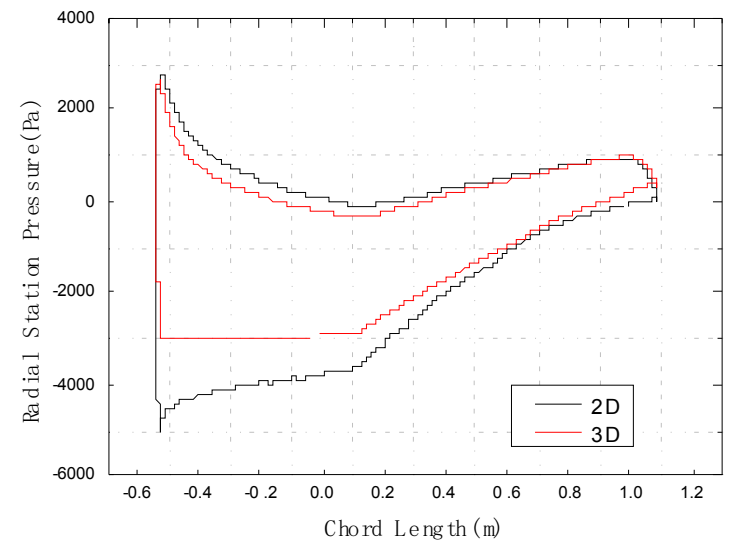

(c)

Figure 6. Streamlines around the radial stations.
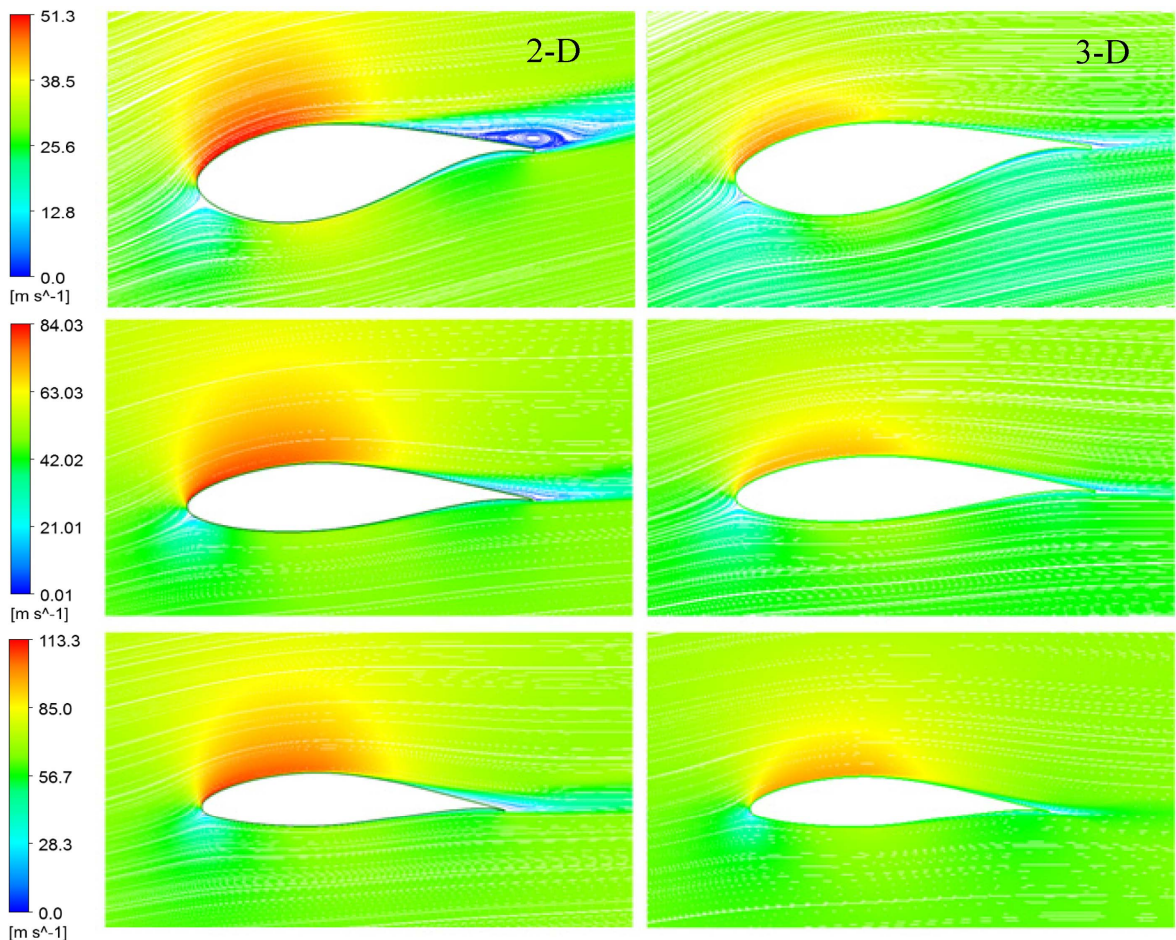

î.
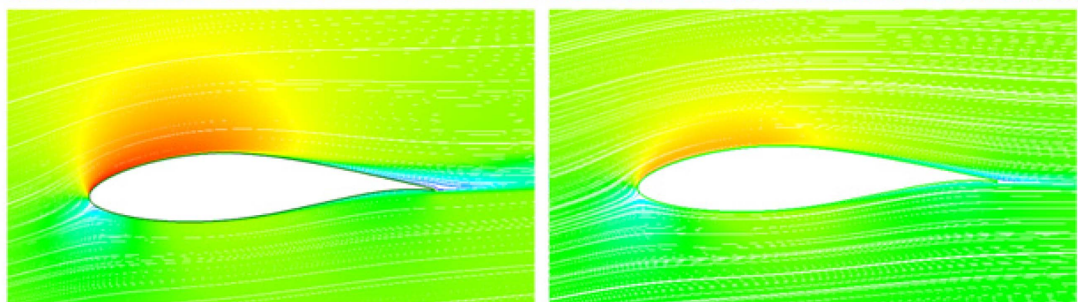

ำ
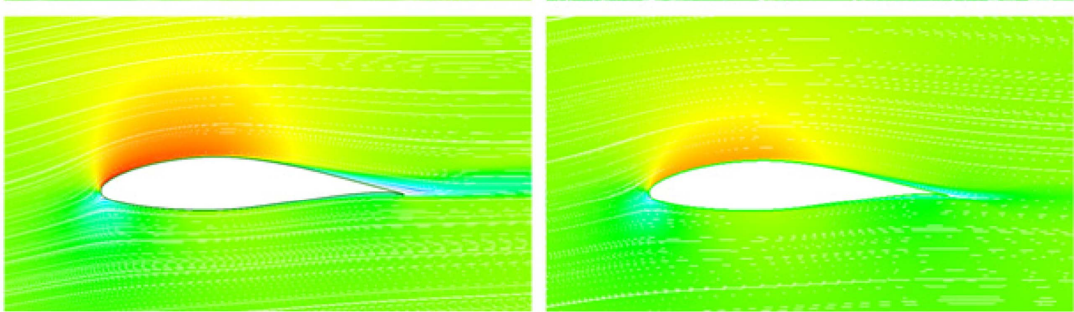

$\underset{\substack{11 \\ 0}}{\substack{1 \\ 0}}$ 
The present wind turbine design approach is typically based on BEM theory. Although the outboard aerodynamic character which is plotted by limiting streamlines on the blade surface in Carcangiu's Ph.D. thesis [16] presents a 2D-alike behavior, the Coriolis and centrifugal forces obviously influence the aerodynamic behavior in the inboard sections. Furthermore, the accurate prediction of rotor blade loads in rotational state is of great importance for sizing the generator and other mechanical components.

\section{Structural Analysis}

\subsection{Finite Element Modeling of the Blade}

The blade geometry is modeled in UG NX as mentioned previously. The finite element model (FEM) is developed in ANSYS software, and there exists a connection functionality which can provide a convenient means to import blade geometry into ANSYS. Before meshing, the blade geometry is divided into a large number of areas in order to define the structural characteristics, including spar caps, shear webs, ply drops, etc. Every time there is a ply drop whatever in the spanwise or chordwise direction, there must be a line to coincide with it because a new laminate definition is required. The materials properties are collected in Table 2 as below.

The structure of the blade is modeled with shell elements (shell99 for all the outer surface and shel191 for the inner shear webs) capable of representing the layer characteristics throughout the shell thickness [17]. After defining the materials lay-up and orientation of the layers of each divided area, the finite-element model is created as Figure $7 \mathrm{a}$ where the mesh density in trailing parts represents the intensively overlapped UD-tapes paralleled to the trailing edge. The final model consists of 80,970 elements and 240,058 nodes. The model total mass reaches $6.13 \mathrm{t}$, which is close to the real blade's weight. To make sure the contact distance is calculated from top surface, the contact offset option should be specify, as shown in Figure $7 \mathrm{~b}$.

Table 2. Material properties for composite blade.

\begin{tabular}{ccccccc}
\hline Material name & Position & E11 $(\mathbf{G P a})$ & E22 $(\mathbf{G P a})$ & $\mathbf{E 1 2}(\mathbf{G P a})$ & Density $\left(\mathbf{k g} / \mathbf{m}^{\mathbf{3}}\right)$ & NUXY \\
\hline UD & Spar & 42.19 & 12.53 & 3.52 & 1910 & 0.24 \\
3AX & Skin & 26.90 & 13.41 & 7.53 & 1910 & 0.47 \\
2AX & Skin & 11.47 & 11.47 & 11.70 & 1909 & 0.614 \\
Balsa Wood & Core & 3.5 & 0.85 & 0.15 & 151 & 0.30 \\
PVC Foam & Core & 0.05 & 0.05 & 0.02 & 60 & 0.085 \\
PET Foam & Core & 0.06 & 0.06 & 0.02 & 110 & 0.085 \\
\hline
\end{tabular}

Figure 7. (a) Finite element of rotor blade; and (b) Blade station of contact offset.

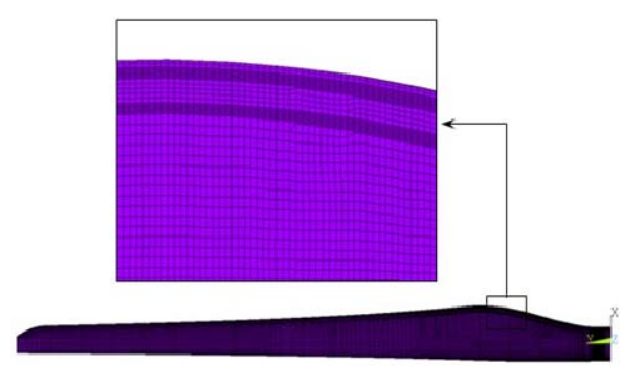

(a)

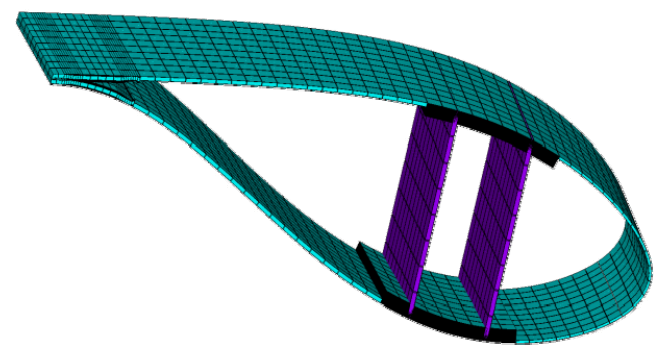

(b) 


\subsection{Dynamic Behavior}

As one of the most complex stress parts of a wind turbine, the blade is under coupled processes of aerodynamic, inertia and elasticity forces. When the natural frequency of blade is the same as the frequency of exciting forces, resonance is produced. To avoid resonance, frequencies must be staggered in the mainly operation intervals. Therefore, the accurate calculates of the nature frequency of blade are of great importance. Usually, these dynamic characteristics are determined for the lowest 3-4 flexural bending modes and for the first torsional mode [18]. The 1.5 MW rotor blade has already been validated by the China Classification Society. The deviation between experimental results and the results from the FE-model is seen in Table 3.

The mode shape results, associated with the computed vibration forms are list in Figure 8.

Apart from the first-order mode, all the computed frequencies are within the adequacy criterion of less than 5\% error. When taking the principal sources of the absolute errors due to the support conditions (boundary conditions) and instrumentation (mass-loading and damping characteristics) [19] into consideration, the FE-modal can be a candidate to predict the rest test observations.

Table 3. Comparison between calculated and natural frequencies.

\begin{tabular}{ccccc}
\hline Mode order & Shape & Calculated frequence (Hz) & Natural frequence (Hz) & Absolute error \\
\hline 1 & flapwise & 1.01 & 0.96 & $5.21 \%$ \\
2 & edgewise & 1.49 & 1.48 & $0.67 \%$ \\
3 & flapwise & 3.02 & 2.88 & $4.86 \%$ \\
4 & edgewise & 4.84 & 4.93 & $1.82 \%$ \\
6 & torsional & 8.42 & 8.40 & $0.23 \%$ \\
\hline
\end{tabular}

Figure 8. Mode shape of blade (a) 1st-flapwise; (b) 2nd-eigewise; (c) 3rd-flapwise; (d) 4th-eigewise; (e) 6th-torsional.

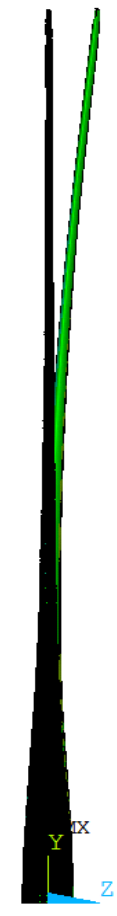

(a)

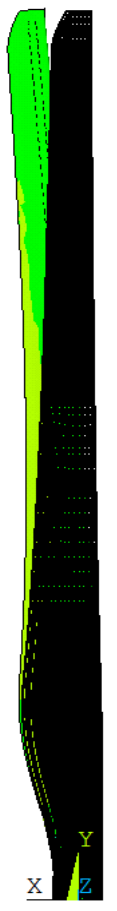

(b)

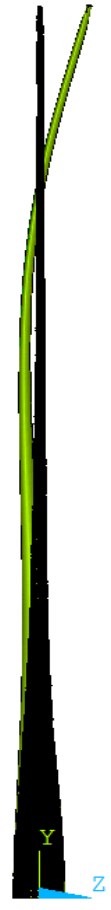

(c)

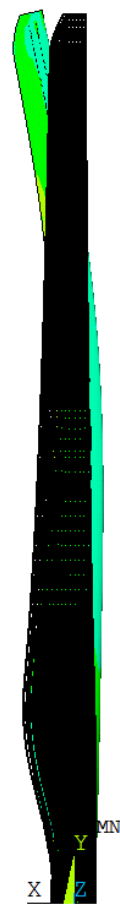

(d)

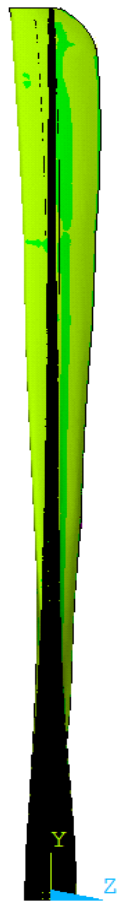

(e) 


\subsection{Static Analysis under Limit Load}

For the structural-response analysis, peak loads are derived by considering a 50 -year extreme gust of $59 \mathrm{~m} / \mathrm{s}$ [14] with the pitch of the blade adjusted to obtain the wind attack-angle associated with the largest aerodynamic loads. The limit CFD loads of the rotor blade are obtained using CFD method mentioned in Section 3.

The latest released ANSYS software provides rapid fluid structure interaction (FSI) simulation. This technique is based on a mesh superposition method in which the fluid and the solid are meshed independently from one another. At first, the SURF154 element is used to overlay onto the outer surface of the FE blade modal. Then, the ANSYS mesh (.cdb) file is generated and imported into CFX-post of extreme gust calculation. Because the geometry of the blade used in CFX and ANSYS is the same one constructed in UG NX, the selected CFX pressure data can be interpolated seamlessly onto the ANSYS surface from the associated ANSYS CFX boundary. The interpolated data is then exported to an ANSYS load file. The FE blade model applied with pressure is shown in Figure 9, where the maximum pressure in the blade tip is $16.126 \mathrm{kPa}$.

Figure 9. FE blade modal applied with limit pressure.

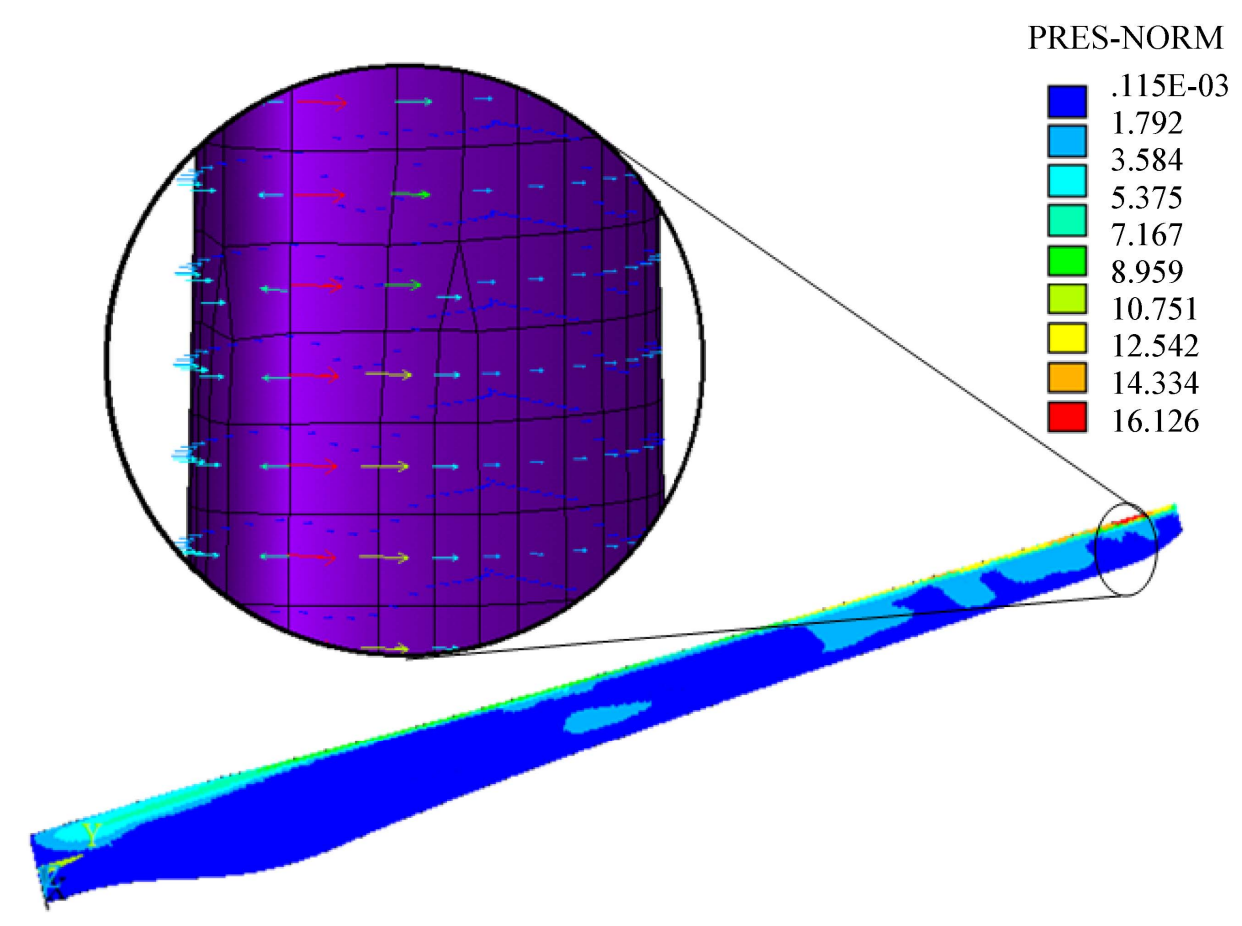

In the present paper, extreme load condition is considered in some detail, while operational load conditions are neglected. When operating under the limit load representing the gust of $59 \mathrm{~m} / \mathrm{s}$, the blade moves into a coupled flapwise and edgewise bending mode. Furthermore, an excessive blade-tip deflection is of major concern in the case of upwind turbine since it might lead to a collision of the blade with the wind turbine tower, and the blade tip deflection determined based on the simulation process is found to be $4.164 \mathrm{~m}$, as seen in Figure 10a, which is less than the rotor overhang, so that the blade is able to withstand extreme wind conditions. However, this value exceeds the recommended maximum allowable value of $0.1 \times R=3.95 \mathrm{~m}$ as mentioned in reference [11], so the blade appears to be stiffness critical. 
Figure 10. (a) The flap-wise deflection of the blade; and (b) The Von Mises stress distribution of the blade.

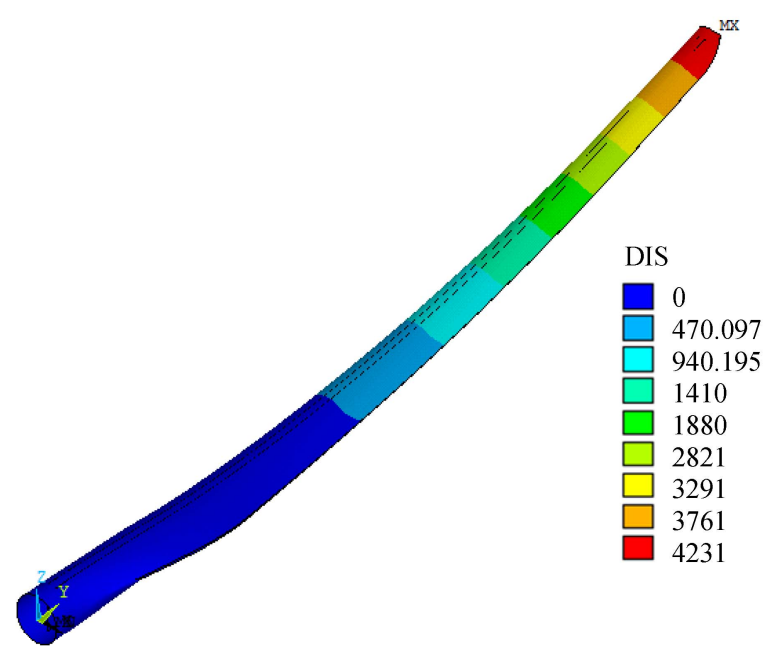

(a)

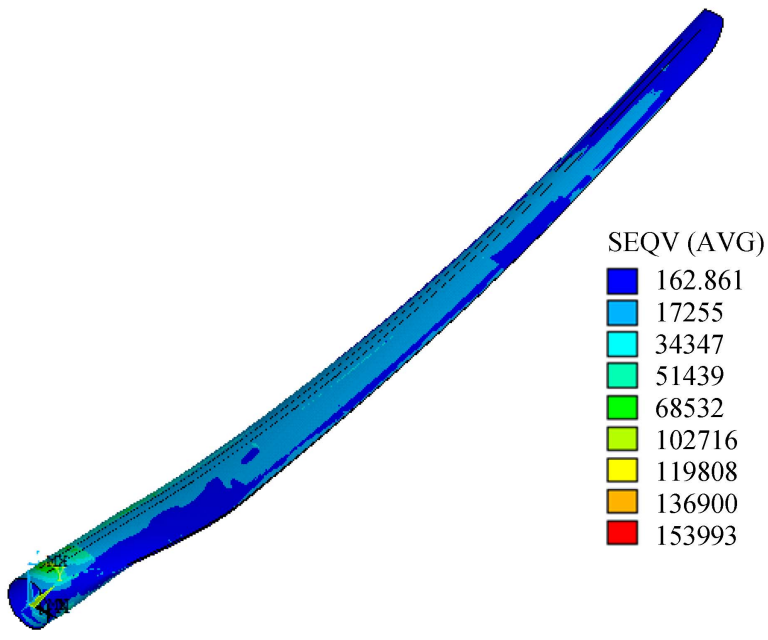

(b)

Static failure of a wind turbine blade can result from exceeding the strength of the material or buckling stability of the structure [20]. The ultimate strength of the blade is estimated by calculating the maximum stress under the extreme wind condition. The ultimate strength is defined by using the ratio of maximum stress $\sigma_{\max }$ to material strength $\sigma_{Y}$ with partial safety factors [21], as seen in Equation (9):

$$
\sigma_{\max } \leq \frac{\sigma_{Y}}{\gamma_{m} \cdot \gamma_{n} \cdot \gamma_{f}}
$$

where the value of $\gamma_{f}$ for this load case is 1.35 ; the value $\gamma_{m}$ for analysis of tensile of compression strength is 1.3 ; and $\gamma_{n}$ for wind turbine class II is 1.15 .

The maximum von Mises stress occurs at the conjunction of the web and spar in the root area is 165.1 $\mathrm{MPa}$ as shown in Figure 10b. However, the provided material strength is $520 \mathrm{MPa}$, the maximum stress calculated with Equation (4) is $257.6 \mathrm{MPa}$, so the blade material can provide enough resistance to extreme winds.

Figure 11 shows the maximum von Mises stress along the trailing web and leading web when the rotor blade are rotating under the wind speed of $59 \mathrm{~m} / \mathrm{s}$ and $11.5 \mathrm{~m} / \mathrm{s}$, respectively. It is observed that the tailing web suffers much larger stress under both wind speed, however, both of the tailing web and leading web are exactly the same, what is more, the two webs are approximate $10 \%$ of the total weight of the rotor blade. In addition, the "stress jump" is due to the alteration of the core materials of the sandwich structure of the webs. Thus, further optimization work on lamination schedule of the webs should be carried out.

Extreme wind loads are deemed the most likely cause of blade buckling. In this paper, the linear eigenbuckling analysis of the 1.5 MW blade is carried out, utilizing the ANSYS software which uses iterative technique to find a set of buckling eigenvalues and eigenvectors to satisfy the follow equation based on the peak loads calculated previously:

$$
\left([K]+\lambda_{i}[S]\right)\{\Psi\}_{i}=\{0\}
$$


where $[K],[S], \lambda_{i}$ and $\{\Psi\}_{i}$ is the stiffness matrix, stiffness matrix, $i$ th eigenvalue (used to multiply the loads which generated $[S]$, also called load factor commonly) and $i$ th eigenvetor of displacements respectively. Figure 12 shows the ANSYS plots of the first five out-of-plane displacement and Table 4 lists the corresponding load factor of the rotor blade.

Figure 11. Maximum von Mises stress along webs.

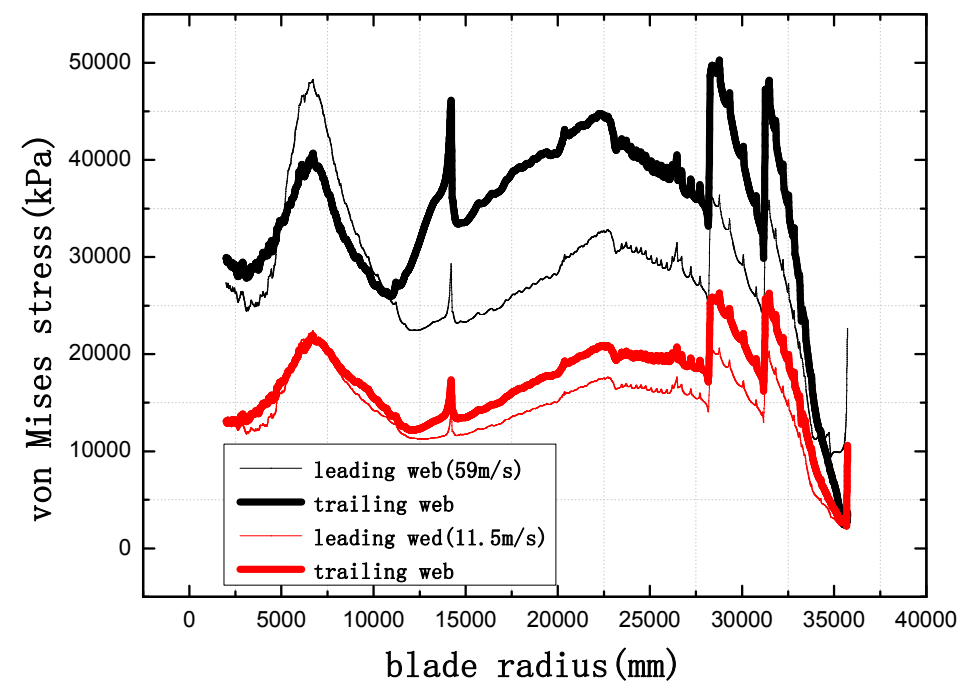

Figure 12. Eigen buckling under limit load (a) 1st mode; (b) 2nd mode; (c) 3rd mode; (d) 4 th mode; (e) 5 th mode. Wind speed $59 \mathrm{~m} / \mathrm{s}$.

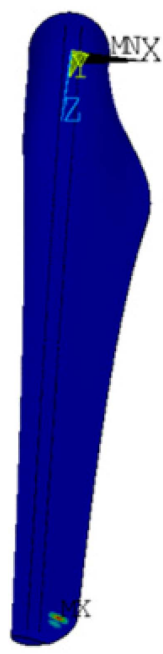

(a)

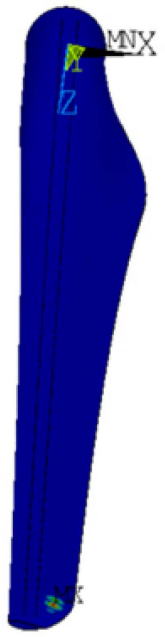

(b)

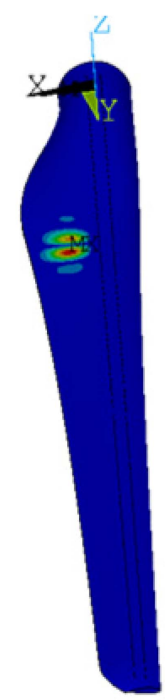

(c)

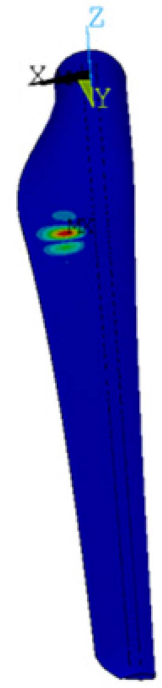

(d)

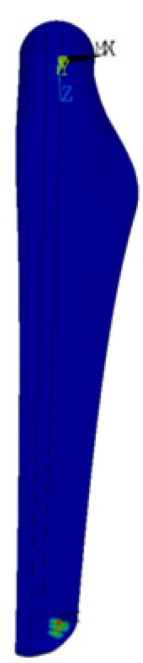

(e)

Table 4. Mode vs. load summary.

\begin{tabular}{ccc}
\hline Model number & Load factor & Location \\
\hline 1st mode (a) & 1.5303 & Tip \\
2nd mode (b) & 1.6779 & Tip \\
3rd mode (c) & 2.2855 & Shoulder \\
4th mode (d) & 2.3458 & Shoulder \\
5th mode (e) & 2.3481 & Tip \\
\hline
\end{tabular}


From the above buckling analysis, the buckling occurs approximately at the tip and shoulder parts of the blade shell when the distributed load approaches or exceeds 1.5303 and 2.2855 times the computed extreme wind load, respectively. The displacement plots in Figure 12 shows a relatively large local inward deformation of both the suction and pressure surfaces of the blade. Following these deformations, either of the two surfaces will find a new stable equilibrium where it can still carry certain aerodynamic loads but much lower than before the deformation point was reached, this is refer to "postbuckling".

\section{Conclusions}

Based on the results obtained in this work, the main conclusions obtained are summarized as follows:

(1) When using the SST turbulence model, the CFD method predicts reasonably well compared with GH-bladed, however, as the wind speed rises, the CFD underestimates the power output. Further work with other turbulence models is required;

(2) At the design point, the BEM method predicts much higher aerodynamic loads than the CFD method while the rotational effect could promote the power output above rated working condition as it stabilizes vortex shedding and limits the growth of the separation layer. It is of great importance for sizing generator and other mechanical with accurate prediction of rotor blade loads in rotational state;

(3) The peak loads derived by considering a 50 -year extreme gust of $59 \mathrm{~m} / \mathrm{s}$ are unidirectionally coupled to the FE-model. The calculated frequencies agree well with the test results. The blade has sufficient flap-wise bending stiffness to maintain the minimal clearance between the blade tip and the turbine tower. The blade material can provide enough resistance to extreme winds, and the buckling would appear when the distributed load exceeds 1.53 times the computed extreme wind load.

\section{Conflict of Interest}

The authors declare no conflict of interest.

\section{References}

1. Manwell, J.F.; Mcgowan, J.G.; Rogers, A.L. Wind Energy Explained, Theory, Design and Application, 2nd ed.; John Wiley and Sons: West Sussex, UK, 2009; pp. 101-121.

2. Curuin, H.D.; Coton, F.N. Validation of a Dynamic Prescribed Vortex Wake Model for Horizontal Axis wind Turbines. In Proceedings of the ASME/JSME 2007 5th Joint Fluids Engineering Conference, Fluids Engineering Division, San Diego, CA, USA, 30 July-2 August 2007; pp. 1069-1075.

3. Duque, E.P.N.; Burklund, M.D.; Johnson, W. Navier-Stokes and comprehensive analysis performance predictions of the NREL phase VI experiment. J. Sol. Energy Eng. 2003, 125, 457-467.

4. Virk, M.S.; Homola, M.C.; Nicklasson, P.J. Effect of rime ice accretion on aerodynamic characteristics of wind turbine blade profiles. Wind Eng. 2010, 34, 207-218. 
5. Keck, R.-E. A numerical investigation of nacelle anemometry for a HAWT using actuator disc and line models in CFX. Renew. Energy 2012, 48, 72-84.

6. Wuchner, R.; Kupzok, A.; Bletzinger, K.U. A framework for stabilized partitioned analysis of thin membrane-wind interaction. Int. J. Mumer. Methods Fluids 2007, 54, 945-963.

7. Jensen, F.M.; Falzon, B.G.; Ankersen, J.; Stang, H. Structural testing and numerical simulation of a 34 m composite wind turbine blade. Compos. Struct. 2006, 76, 52-61.

8. Hermann, T.M.; Mamarthupatti, D.; Locke, J.E. Postbuckling analysis of a wind turbine blade substructure. J. Sol. Energy Eng. 2005, 127, 262-280.

9. Rajadurai, J.S.; Christopher, T.; Thanigaiyarasu, G.; Nageswara Rao, B. Finite element analysis with an improved failure criterion for composite wind turbine blades. Forsch. Ing. 2008, 72, 193-207.

10. Grujicic, M.; Arakere, G.; Sellappan, V.; Vallejo, A.; Ozen, M. Structural-response analysis, fatigue-life prediction and material selection for $1 \mathrm{MW}$ horizontal-axis wind-turbine blades. J. Mater. Eng. Perform. 2010, 19, 780-801.

11. Grujicic, M.; Arakere, G.; Pandurangan, B.; Sellappan, V.; Vallejo, A.; Ozen, M. Multidisciplinary optimization for fiber-glass reinforced epoxy-matrix composite $5 \mathrm{MW}$ horizontal-axis wind-turbine blades. J. Mater. Eng. Perform. 2010, 19, 1116-1127.

12. Sutherland, H.J. On the Fatigue Analysis of Wind Turbines; SANDA99-0089; Sandia National Laboratories: Albuquerque, NM, USA, 1999.

13. Menter, F.R.; Kuntz, M.; Langtry, R. Ten years of industrial experience with the SST turbulence model. Heat Mass Transf. 2003, 4, 625-632.

14. International Electrotechnical Commission. IEC International Standard 61400-1, Part I: Design Requirements, 3rd ed.; International Electrotechnical Commission: Geneva, Switzerland, 2005.

15. Cimbala, J.M. Introduction to Computational Fluid Dynamics, 2nd ed.; Penn State University: University Park, PA, USA, 2012.

16. Carcangiu, C.E. CFD-RANS Study of Horizontal Axis Wind Turbines. Ph.D. Thesis, University of Cagliari, Cagliari, Italy, 2008.

17. McKittrick, L.R.; Cairns, D.S.; Mandell, J.; Combs, D.C.; Rabern, D.A.; VanLuchene, D.V. Analysis of a Composite Blade Design for the AOC 15/50 Wind Turbine Using a Finite Element Model; SAND 2001-1441; Sandia National Laboratories: Albuquerque, NM, USA, 2001.

18. Larsen, G.C.; Hansen, M.H.; Baumgart, A.; Carlén, I. Modal Analysis of Wind Turbine Blades; Risø National Laboratory: Roskilde, Denmark, 2002.

19. Todd, G.D.; Carne, T. Experimental modal analysis of research-sized wind turbine blades. Sound Vib. 2010, 44, 8-11.

20. Bir, G.; Migliore, P. A Computerized Method for Preliminary Structural Design of Composite Wind Turbine Blades; AIAA-2001-0022; National Wind Technology Center, National Renewable Energy Laboratory: Golden, CO, USA, 2001.

21. Hu, W.F.; Han, I.; Park, S.C.; Choi, D.H. Multi-objective structural optimization of a HAWT composite blade based on ultimate limit state analysis. J. Mech. Sci. Technol. 2012, 26, 129-135.

(C) 2013 by the authors; licensee MDPI, Basel, Switzerland. This article is an open access article distributed under the terms and conditions of the Creative Commons Attribution license (http://creativecommons.org/licenses/by/3.0/). 